\title{
Magnetic-Fluorescent Colloidal Nanobeads: Preparation and Exploitation in Cell Separation Experiments ${ }^{a}$
}

\author{
Riccardo Di Corato, Philomena Piacenza, Mariarosaria Musarò, \\ Raffaella Buonsanti, Pantaleo Davide Cozzoli, Massimo Zambianchi, \\ Giovanna Barbarella, Roberto Cingolani, Liberato Manna, Teresa Pellegrino*
}

Nanostructures displaying fluorescence and magnetic properties at the same time are potentially useful for achieving simultaneous bio-separation and bio-sensing (e.g., magnetic separation coupled with multiplexing optical detection of different tumour cell populations). Spherical nanobeads that display both fluorescent and magnetic features are reported; they are fabricated by grafting fluorescent oligothiophene molecules to an amphiphilic polymer that is then used to enwrap iron oxide nanoparticles, which acts as the magnetic domain. By tuning experimental conditions, control over the number of magnetic nanoparticles per bead and over the bead diameter $(30-400 \mathrm{~nm})$ was achieved. A cell separation efficiency of the level required for cell sorting applications is also reported.

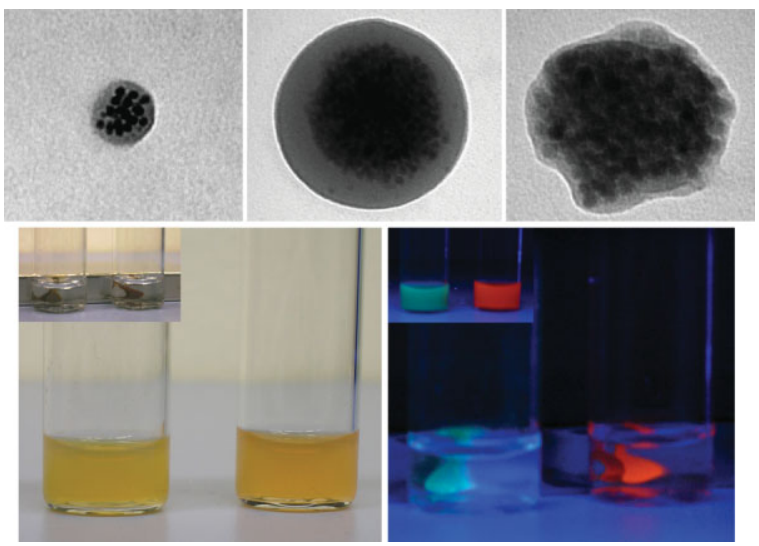

\section{Introduction}

The success of cancer therapy depends on multiple factors, among which an early and accurate diagnosis is fundamental. Often the cancer lesion is identified only at an

R. Di Corato, P. Piacenza, M. Musarò, R. Buonsanti, P. D. Cozzoli, T. Pellegrino

National Nanotechnology Laboratory of CNR-INFM and IIT Research Unit, via per Arnesano Km 5, 73100 Lecce, Italy Fax: +390832 298230; E-mail: teresa.pellegrino@unisalento.it R. Di Corato, R. Buonsanti, P. D. Cozzoli

Scuola Superiore ISUFI, Università del Salento, Distretto Technologico, via per Arnesano Km 5, 73100 Lecce, Italy G. Barbarella

Mediteknology s.r.l., Area della Ricerca CNR, ISOF, I-40 I-40129 Bologna, Italy advanced stage of the disease, mainly due to the absence of a highly sensitive technique capable of detecting low levels of tumour cells and/or markers in biological samples. Scientists are tackling this issue by reducing the probe size down to the nanoscale, which is expected to increase the

M. Zambianchi

CNR- ISOF I-4O I-40129 Bologna, Italy

L. Manna, R. Cingolani

Fondazione Istituto Italiano di Tecnologia, Via Morego 30, 16163 Genova, Italy

\footnotetext{
$\mathrm{a}$. Supporting information for this article is available at the bottom of the article's abstract page, which can be accessed from the journal's homepage at http://www.mbs-journal.de, or from the author.
} 
detection sensitivity due to the higher surface-tovolume ratio. $^{[1,2]}$

A nanosystem designed for bio-separation must satisfy the basic requirement of being able to accumulate promptly via a magnet (so that a pre-accumulation step is possible), while its size must be small enough to ensure a high sensitivity. Also, it must be easily dispersible in the medium in which it has to be exploited and to interact with cells or markers. A system designed for multiplexing detection must additionally include code molecules (i.e., fluorophores) within its structure so as to be able to distinguish in parallel various analytes and/or tumour cells in the same biological sample. Multifunctional nanotools able to perform detection and separation tasks simultaneously, which have been reported recently, have been based mainly on assemblies of magnetic nanoparticles and quantum dots. ${ }^{[3-7]}$ Although most such nanostructures can be prepared with sizes smaller than $200 \mathrm{~nm}$, the fabrication methods by which they are made do not allow systematic tuning of their dimensions down to a few tens of nanometers and/or the control over the resulting bead morphology (for instance the number of iron oxide nanoparticles incorporated in each bead, which in turn affects their feature of being magnetically attracted). Simultaneous control over the bead morphology and size would allow design of magnetic beads with well-defined magnetic properties for application both in vivo, such as for drug delivery, and in vitro, as for instance in cell sorting experiments.

Here, we report the fabrication of magnetic-fluorescent nanobeads (MFNBs) in a size range between 30 and $400 \mathrm{~nm}$. They are based on aggregates of iron oxide $\left(\gamma-\mathrm{Fe}_{2} \mathrm{O}_{3}\right)$ nanoparticles (IONPs) enwrapped within an amphiphilic polymer, [namely poly(maleic anhydride-alt-1-octadecene), $\mathrm{P}_{\mathrm{C} 18}$ ] to which oligothiophene fluorescents (OTFs) are grafted. We demonstrate fine control over the solutionphase clustering of IONPs and $\mathrm{P}_{\mathrm{C} 18}$, which enables us to prepare MFNBs for which we can tune both the size and the radial distribution of IONPs in their interior.

We chose OTFs because they represent a viable alternative as fluorescent labels to traditional organic dyes in biomedical applications. Indeed, OTFs are characterized by structure-dependent emission spectra (closely related to the number of thiophene rings and to the type of substituents thereon), high photochemical stability, and low toxicity. In addition, OTFs exhibit broad excitation spectra, similar to quantum dots, ${ }^{[8,9]}$ which potentially allows for easier multiplexing analysis due to the possibility to excite various types of OTFs (each emitting at a different colour) with a single light wavelength. ${ }^{[10]}$

IONPs were chosen as the magnetic material because of their superparamagnetic properties and biocompatibility. Several groups have assembled IONPs into clusters of a few hundreds of nanometers in size, and these displayed a faster response to an external magnet than the starting individual
IONPs in solution. ${ }^{[5,11-13]}$ Clustering is desired as IONPs smaller than $15 \mathrm{~nm}$ exhibit low magnetization that prevents both their accumulation within a few minutes under application of moderate magnetic fields (i.e., $<0.3 \mathrm{~T}$ ) and control of their motion when they are dispersed in biological liquids, like blood. The polymer $\mathrm{P}_{\mathrm{C} 18}$ used in this work to prepare the beads has been recently exploited to solubilize several types of nanoparticles in water ${ }^{[14]}$ and to prepare quantum dot barcode nanobeads for multiplexing detection. ${ }^{[2]}$ Herein, we demonstrate that our newly developed MFNBs prepared by employing the $\mathrm{P}_{\mathrm{C} 18}$ polymer displayed both a rapid response to a magnet and a highly stable and tuneable fluorescent signal. Moreover, in cell separation tests, the beads exhibited high efficiency (up to $100 \%$ ) in the separation of tumour cells in suspension within $30 \mathrm{~min}$.

\section{Experimental Part}

\section{Preparation of Beads}

We describe here the general procedure devised to prepare magnetic nanobeads. To $8 \mu \mathrm{L}$ of a solution of $\mathrm{P}_{\mathrm{C} 18}$ in tetrahydrofuran (THF) $\left(50 \times 10^{-3} \mathrm{M}\right.$, this concentration is expressed in terms of polymer monomer units), $20 \mu \mathrm{L}$ of a $2 \times 10^{-6} \mathrm{M}$ solution of IONPs (13 nm in diameter) in THF was added. An amount of THF was then added to this solution to reach a final volume of $200 \mu \mathrm{L}$. The mixture was shaken for 30 min on a vortex system at $1000 \mathrm{rpm}$ (we avoided use of a magnetic stirrer bar since the beads tended to stick to it). Subsequently, $800 \mu \mathrm{L}$ of acetonitrile (ACN) was added at a rate of $0.25 \mathrm{~mL} \cdot \mathrm{min}^{-1}$. Alternative solvents, such as water, acetone, methanol, $\mathrm{N}, \mathrm{N}$-dimethylformamide (DMF), or dimethyl sulfoxide (DMSO), could be used. In such cases, a solvent volume of $800 \mu \mathrm{L}$ was added at a rate of $0.25 \mathrm{~mL} \cdot \mathrm{min}^{-1}$. After the beads were formed, a magnet (NdFeB, $0.3 \mathrm{~T}$ ) was positioned close to one side of the vial, which led to quantitative accumulation of the beads to that side of the vial. The mother solution in the vial was then replaced with a borate phosphate buffer $\left(50 \times 10^{-3} \mathrm{M}, \mathrm{pH} 9\right)$ and the beads were redissolved in the buffer upon removal of the magnet. The beads prepared by addition of $\mathrm{ACN}$ were composed of a core of aggregated particles encased by a shell of polymer. The thickness of the polymer shell in these beads (Figure $1 \mathrm{c}$ ) was related the volume of $\mathrm{P}_{\mathrm{C} 18}$ polymer solution $\left(50 \times 10^{-3} \mathrm{M}\right.$ in THF) added to the solution of IONPs, which could be $4,8,12$, or $16 \mu \mathrm{L}$.

In general, the critical parameters that controlled bead formation were the speed at which the polymer and the IONPs were mixed, the mixing time needed before the addition of the solvent of different polarity, and the rate at which such solvent was added.

\section{$\mathrm{NH}_{2}-\mathrm{OTFs}$ and NHS-OTFs Functionalization}

To equip the magnetic nanobeads with OTFs, two approaches were followed. In the first approach, we employed OTF derivates based on amino-functionalized molecules $\left(\mathrm{NH}_{2}-\mathrm{OTF1}\right.$ ) (Figure $2 \mathrm{a}$ ), which were grafted to the $\mathrm{P}_{\mathrm{C} 18}$ polymer. In detail, to a solution of $\mathrm{NH}_{2}-$ 

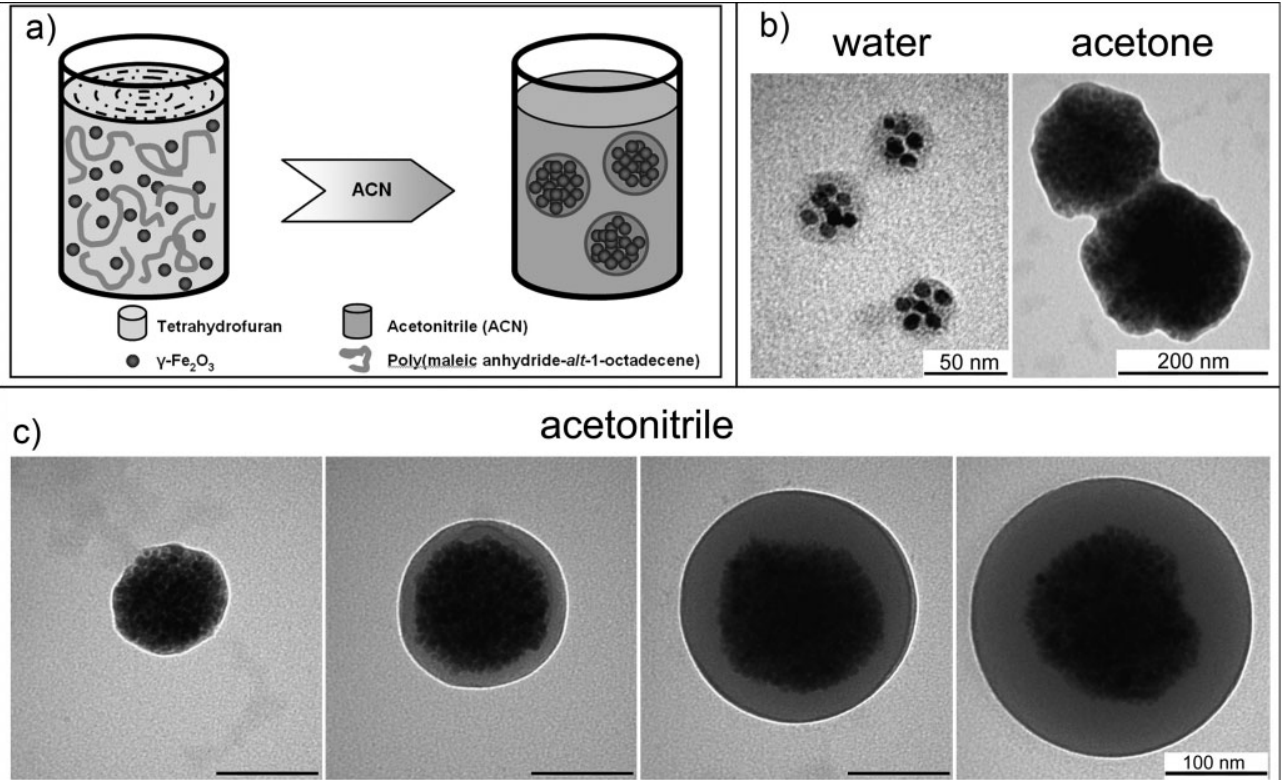

acetonitrile

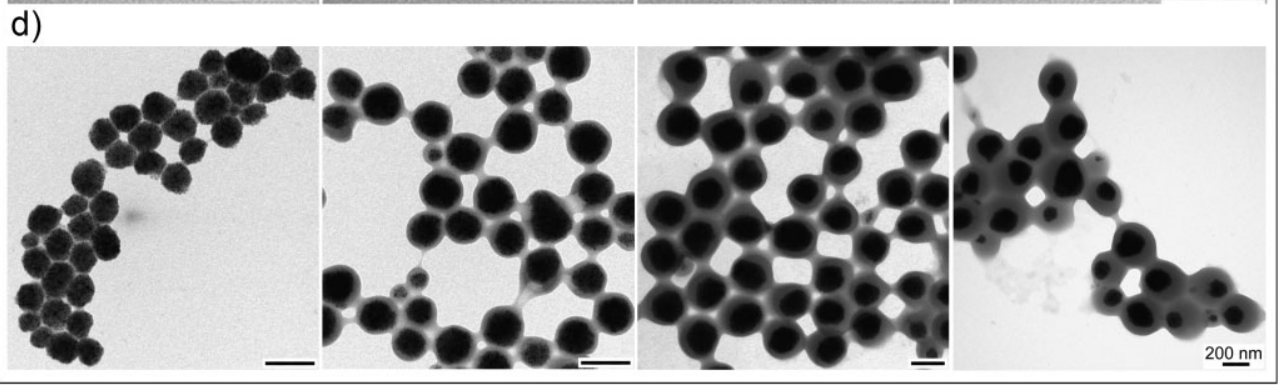

Figure 1. a) Sketch of the procedure devised to synthesize the beads. Adding a solvent like acetonitrile (ACN) to a solution of $\mathrm{P}_{\mathrm{C} 18}$ and hydrophobic iron oxide $\left(\gamma-\mathrm{Fe}_{2} \mathrm{O}_{3}\right)$ nanoparticles (IONPs) in THF, at a controlled flow rate, promotes formation of the beads. b) Beads prepared by addition of water (left) and acetone (right). c) TEM images of single beads of different diameters obtained by addition of acetonitrile, at increasing concentrations of polymer $\left(1,2,3\right.$, and $4 \times 10^{-3} \mathrm{M}$, respectively). d) Corresponding low-magnification TEM overviews of beads shown in panel $c$.

OTF1 ${ }^{[15]}$ in THF $\left(5 \times 10^{-3} \mathrm{M}\right)$ a solution of $\mathrm{P}_{\mathrm{C} 18}$ in THF $\left(50 \times 10^{-3} \mathrm{M}\right)$ was added. Then an amount of THF was added to reach a final volume of $200 \mu \mathrm{L}$ and the solution was shaken at room temperature overnight. The resulting mixture was added to a dried powder of $\gamma$ $\mathrm{Fe}_{2} \mathrm{O}_{3}$ nanoparticles (obtained by drying $20 \mu \mathrm{L}$ of a $2 \times 10^{-6} \mathrm{M}$ solution of IONPs) and shaken at $1000 \mathrm{rpm}$ for $30 \mathrm{~min}$, after which $800 \mu \mathrm{L}$ of $\mathrm{ACN}$ was added at a rate of $0.25 \mathrm{~mL} \cdot \mathrm{min}^{-1}$. In a series of experiments, different volumes of the $\mathrm{NH}_{2}-\mathrm{OTF} 1$ solution $(1,10,20$, 50, and $100 \mu \mathrm{L}$, respectively) were mixed with $10 \mu \mathrm{L}$ of the $\mathrm{P}_{\mathrm{C} 18}$ polymer solution in order to reach well-defined ratios of $\mathrm{NH}_{2}-\mathrm{OTF} 1$ molecules added every 100 polymer units $(1,10,20,50$, and 100, respectively).

In the second approach, we employed OTF derivates bearing an $\mathrm{N}$-hydroxysuccinimidyl group (henceforth referred to as NHS-OTFs or NHS-OTF1 and NHS-OTF2). ${ }^{[10]}$ These were also grafted to the $\mathrm{P}_{\mathrm{C} 18}$ polymer. First, $20 \mu \mathrm{L}$ of a NHS-OTF solution in THF $\left(5 \times 10^{-3} \mathrm{M}\right)$ were mixed with $20 \mu \mathrm{L}$ of a solution of bis(hexamethylene)triamine in THF $\left(5 \times 10^{-3} \mathrm{M}\right)$. Then an amount of THF was added to reach a final volume of $190 \mu \mathrm{L}$ and the solution was vortexed overnight. The resulting mixture was reacted with $10 \mu \mathrm{L}$ of $\mathrm{P}_{\mathrm{C} 18}$ in THF $\left(50 \times 10^{-3} \mathrm{M}\right)$ and left under vortexing for an additional $12 \mathrm{~h}$ at room temperature. The reaction mixture was finally added to a dried powder of IONPs (prepared by drying $20 \mu \mathrm{L}$ of a $2 \times 10^{-6} \mathrm{M}$ solution of IONPs) and to this solution $800 \mu \mathrm{L}$ of ACN was added at a rate of $0.25 \mathrm{~mL} \cdot \mathrm{min}^{-1}$.

In both approaches described above, for purification of the MFNBs from excess OTF derivates, the bead solution was loaded in a $10 \%$ sucrose solution and centrifuged at $3000 \mathrm{~g}$ for $2 \mathrm{~h}$ in $12-\mathrm{mL}$ tubes. The OTF derivates lay in the upper part of the sucrose solution, while the beads were recovered from the bottom of the tube by applying a magnet to the vial wall. The sucrose solution was replaced with a solution of $50 \times 10^{-3} \mathrm{M}$ borate buffer ( $\mathrm{pH}$ 9).

\section{Results and Discussion}

To prepare the beads, surfactant-coated IONPs, which were synthesized by a non-hydrolytic route based on modified literature protocols ${ }^{[16]}$ (see Supplementary Information for more details), were suspended in THF and mixed with a THF solution of $\mathrm{P}_{\mathrm{C} 18}$ under vigorous shaking. To the latter 


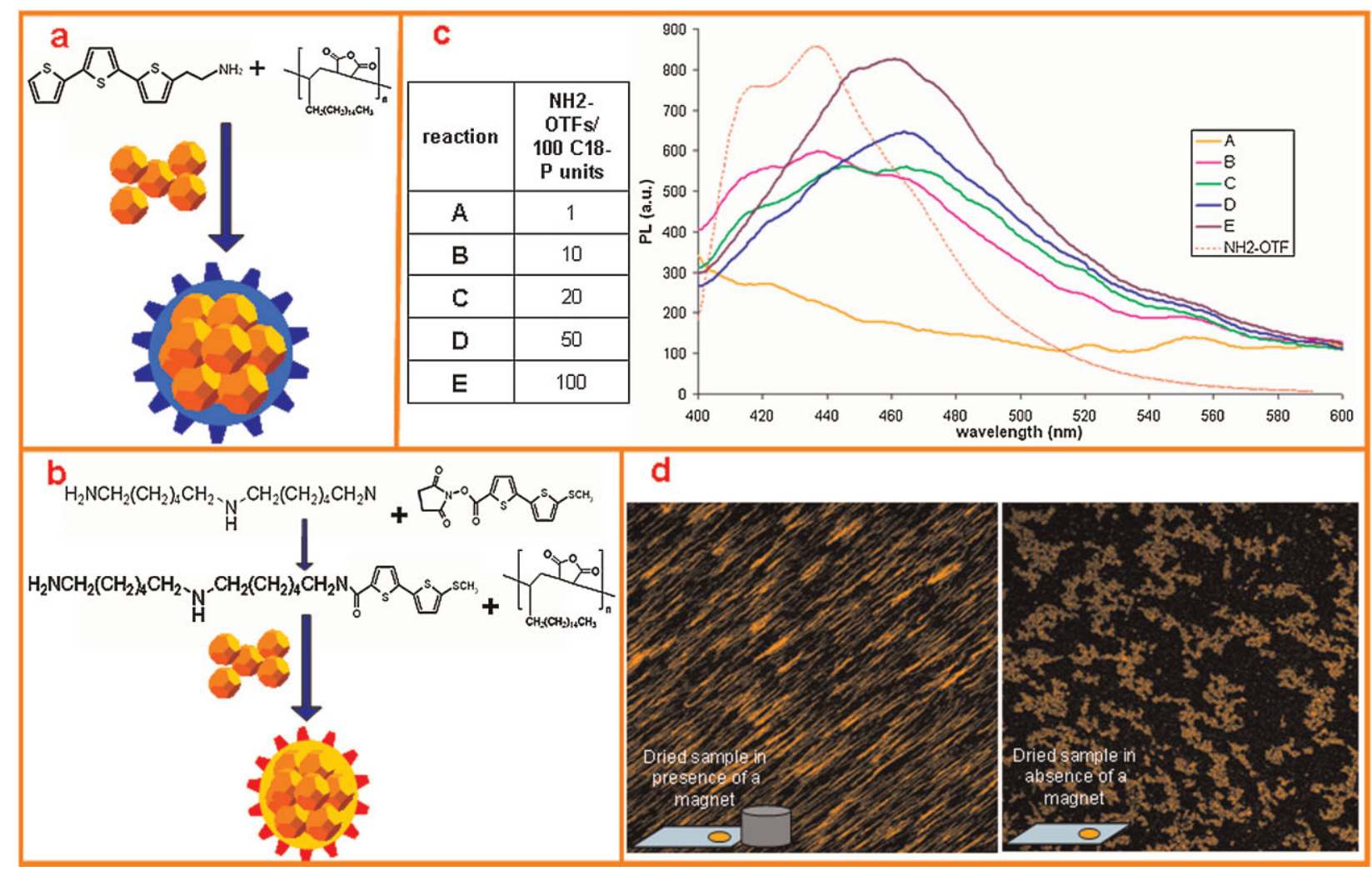

Figure 2. a) Scheme of the magnetic-fluorescent nanobead (MFNB) formation by exploiting $\mathrm{NH}_{2}$-oligothiophene fluorescents ( $\mathrm{NH}_{2}-\mathrm{OTFs}$ ). The $\mathrm{NH}_{2}$-OTF1s (left) are grafted to the polymer chain via reaction with the anhydride groups. b) Scheme of MFNB formation by using $N$ hydroxysuccinimidyl-OTFs (NHS-OTFs). The first step involves the reaction of NHS-OTF1 (right) to diamino linker, followed by grafting of the amino linker-OTF conjugate to the polymer. c) PL spectra of MFNBs functionalized with different amounts of $\mathrm{NH}_{2}-\mathrm{OTF}$. By increasing the number of molecules of $\mathrm{NH}_{2}-\mathrm{OTF} 1$ grafted to the polymer, an increased fluorescent signal for MFNBs was recorded. d) Confocal study of the influence of the magnetic field on the assembly of MFNBs. A drop of MFNBs was dried on a glass slide in the presence of a NdFeB magnet (left). A control experiment was performed by drying a drop of sample in the absence of the magnet (right).

mixture, a solvent was added in which the IONPs were not soluble and the solubility of $\mathrm{P}_{\mathrm{C} 18}$ was altered, such that a controlled aggregation of the nanoparticles and wrapping of the polymer chains were simultaneously induced, yielding polymer beads with IONP aggregates inside (Figure 1a). The beads were temporarily attracted to a magnet, allowing for removal of the mixture of solvents in which they were dissolved, following which they were redissolved in borate buffer solution $\left(50 \times 10^{-3} \mathrm{M}, \mathrm{pH} 9\right)$. We investigated the parameters that induce the formation of the beads and that regulate their size and the radial distribution of IONPs at their interior. The latter was dictated mainly by the solubility of the polymer $\mathrm{P}_{\mathrm{C} 18}$ in the solvent added to the THF solution and by the concentration of polymer (Figure 1b,c). Adding a solvent in which the polymer is completely insoluble (i.e., water) yielded small beads (32 $\pm 8 \mathrm{~nm}$ ), each embedding a few IONPs (Figure $1 \mathrm{~b}$, left image). On the other hand, adding a solvent in which the polymer is fully soluble (e.g., acetone) yielded beads embedding a higher number of IONPs (Figure $1 \mathrm{~b}$, right image). In the latter case, however, we achieved a poor control of the size distribution of the beads (average size $139 \pm 62 \mathrm{~nm}$ ). Similar results were found when adding DMF, a solvent in which the polymer has solubility similar to that in acetone (see Supplementary Figure S1).

On the other hand, the addition of a solvent in which the polymer is partially soluble, such as ACN or DMSO, led to the formation of magnetic beads in a broader range of conditions. When adding $\mathrm{ACN}$, the beads were characterized by a nucleus of aggregated particles encased by an outer polymer shell (Figure 1c,d). A thicker polymer shell was formed at higher concentrations of polymer in the starting THF solution (Figure 1c). Interestingly, an outer shell was observed even at the lowest concentration of polymer used $\left(1 \times 10^{-3} \mathrm{M}\right.$, Figure $1 \mathrm{c}$, first image from the left). The total bead size (core of aggregated particles + polymer shell) could be varied between 100 and $400 \mathrm{~nm}$, and the size of the core of aggregated particles could be varied between 100 and $170 \mathrm{~nm}$ (see Supplementary Figure S2). In general, we observed that a thicker 
polymer shell led to the resulting beads being more stable in water (the most stable beads did not precipitate even in a $\mathrm{NaCl}$ concentration as high as $0.5 \times 10^{-3} \mathrm{M}$, in a pH range between 4 and 10).

The importance of the solubility parameters of the polymer, not only for the formation of the beads, but also for controlling their size, was assessed by control experiments in which the beads were prepared in the absence of IONPs. It was found that polymer beads were formed if a solvent like ACN was used, in which the polymer is nearly insoluble, which indicates the rearrangement of the amphiphilic polymer in solution promoted by the change in polarity of the starting solution (see Supplementary Figure S1 and S3). Similar results were found with solvents having similar solubility parameters for $\mathrm{P}_{\mathrm{C} 18}$, such as DMSO or methanol (see Supplementary Figure S1). The beads were formed even in water, in which $\mathrm{P}_{\mathrm{C} 18}$ is completely insoluble. The beads did not form either in acetone or DMF, in which $\mathrm{P}_{\mathrm{C} 18}$ is indeed soluble (see Supplementary Figure S1 and S4). Control experiments were also carried out using only IONPs in the absence of polymer. In this case even if the IONPs started to organize in small two-dimensional clusters, as seen by transmission electron microscopy (TEM) (see Supplementary Figure S5), these aggregates were neither stable nor soluble in water, and therefore no beads were observed.

Taken together the results above highlight the important role of the amphiphilic polymer in the reaction mixture. The change in polarity of the solution upon addition of the solvent induces a conformational rearrangement of $\mathrm{P}_{\mathrm{C} 18}$ and simultaneously promotes hydrophobic interactions among surfactant-coated IONPs, as well as between the alkyl chains of $\mathrm{P}_{\mathrm{C} 18}$ polymer and the organic-capped IONPs. These concerted interactions lead to the formation of the beads. Furthermore the polymer equips the bead surface with carboxylic groups, derived from partial hydrolysis of the anhydrides present on the polymer chains (determined by FT-IR, see Supplementary Figure S6). The negative charges introduced at the bead surface make them soluble in polar solvents like ethanol or water. The prepared beads moved to the magnet within a few minutes depending on their sizes (e.g., $5 \mathrm{~min}$ for $120 \mathrm{~nm}$ beads).

A further step in designing the beads concerned their functionalization with fluorescent molecules, which makes them suitable for both separation and detection. To this aim we first grafted the OTFs to the $\mathrm{P}_{\mathrm{C} 18}$ polymer and subsequently applied the procedure described above to prepare the beads. For coupling of the OTFs to $\mathrm{P}_{\mathrm{C} 18}$, we exploited the anhydride groups of the polymer chain, which were opened upon reaction with $\mathrm{NH}_{2}-\mathrm{OTF} 1 s$ (Figure 2a). After mixing $\mathrm{P}_{\mathrm{C} 18}$ with a solution of $\mathrm{NH}_{2}-\mathrm{OTFs}$ in THF and stirring overnight at room temperature, the reaction products were characterized by gel electrophoresis, which indicated the successful grafting of the OTFs to $\mathrm{P}_{\mathrm{C} 18}$ (see
Supplementary Figure S7). This mixture was then added to dry IONPs and the beads were prepared following the procedure described above. In this case, to ensure removal of free OTF derivates, a centrifugation step in a sucrose solution was performed (see Supplementary Figure S8).

Different ratios of OTF molecules per polymer chain were tested, and the photoluminescence (PL) spectra of the cleaned beads were recorded. A higher fluorescent signal was observed by increasing the number of OTFs per 100 polymer monomer units (Figure $2 \mathrm{~b}$ and see Supplementary Figure S9). The fluorescent peak of the conjugates was redshifted with respect to that of the free OTFs, and the higher the number of OTFs added to the polymer, the larger the shift was. Even the absorption spectrum at higher concentrations of OTFs in the polymer bead was modified with respect to that of free OTF (see Supplementary Figure S10). Both features are likely to be a consequence of a structural conformation change of the OTFs in the beads. TEM analysis indicated that the shape of the beads was governed by the number of OTFs incorporated per bead, and that the beads had narrow size dispersions (see Supplementary Figure S11). These findings were supported by dynamic light scattering (DLS), by which we identified size dispersions below 20\% (see Supplementary Figure S12).

The procedure was also successful for OTFs bearing an $N$ hydroxysuccinimidyl group (NHS-OTFs) as a functional group. In this case, an earlier amino functionalization of the OTFs was required (Figure $2 \mathrm{~b}$ ). A diamino linker was first bound on one side of the NHS-OTF; then on the other side it was reacted with the anhydride ring of $\mathrm{P}_{\mathrm{C} 18}$, as described above. In this case, PL and TEM analyses confirmed the formation of the beads and the retention of the fluorescence (see Supplementary Figure S13), while the successful reaction between the OTFs and the diamino linker and finally with the polymer was proven by gel electrophoresis (see Supplementary Figure S14).

To check at the same time the fluorescence of the MFNBs and their response to a magnetic field, a drop of the sample was deposited onto a substrate and allowed to dry in the presence of a magnet, after which it was imaged by a confocal microscope. The MFNBs were organized in fluorescent stripe patterns aligned along the magnetic force lines, indicating that the beads could move on the substrate during evaporation via a strong interaction with the magnetic field (such patterns were obviously not observed in the absence of a magnet) (Figure 2d).

In order for the MFNBs to be applied in cell sorting experiments, one needs to demonstrate their ability to perform cell separation before any specific targeting functionalization of their surface is done. To this aim, magnetic beads of about $120 \mathrm{~nm}$ in diameter at different concentrations, measured as total amount of iron added $\left(6.5,16,32.5\right.$, and $65 \mu \mathrm{g} \cdot \mathrm{mL}^{-1}$, respectively), were added to $\mathrm{KB}$ tumour cell cultures and cultured for $24 \mathrm{~h}$ at $37^{\circ} \mathrm{C}$. 


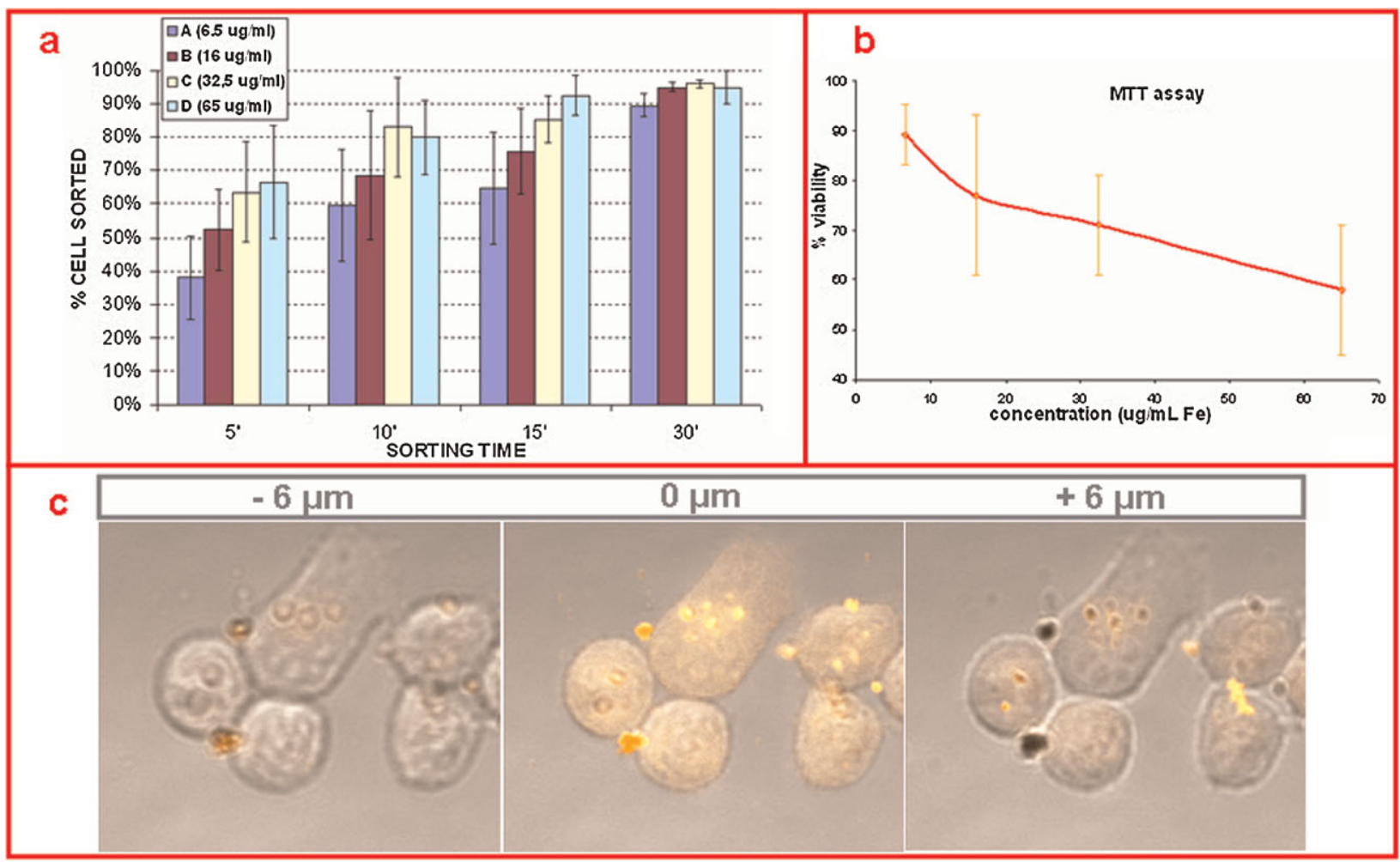

Figure 3. a) Results of cell separation experiments. KB cells doped with four concentrations of MFNBs $\left(6.5,16,32.5\right.$, and $65 \mu \mathrm{g} \cdot \mathrm{mL}^{-1}$ of iron) were accumulated to the magnet at 5, 10,15, and $30 \mathrm{~min}$. The counted cells are reported versus the incubation time. b) Cell viability test on KB cells doped with increased amounts of MFNBs. c) Sections of KB cells doped with MFNBs based on OTF2.

Simple cell separation tests were performed on the doped cells: the adherent cells were detached, counted and redissolved in a known volume of medium. Subsequently, a magnet was applied to the vial containing the cells for different times $(5,10,15$, and $30 \mathrm{~min}$, respectively), and after the time lapse the cells close to the magnet were collected separately from the fraction of cells in the medium. The statistical results of cells counted at different times, doped with different amounts of magnetic beads, are reported in Figure 3a. When doping the cells with increasing amounts of MFNBs, a larger number of cells were collected to the magnet, while for a given concentration of MFNBs in the cells, increasing the time the magnet was applied resulted in a larger number of cells being collected. In the best case, cells doped with $65 \mu \mathrm{g} \cdot \mathrm{mL}^{-1}$ of iron were almost quantitatively separated (95\%) from the solution within the first $30 \mathrm{~min}$. Even when the cells were doped with the lowest concentration of iron $\left(6.5 \mu \mathrm{g} \cdot \mathrm{mL}^{-1}\right)$, a separation efficiency of $65 \%$ was found after $30 \mathrm{~min}$.

We compared our data on cell accumulation with those reported in our previous study ${ }^{[10]}$ in which single IONPs functionalized with OTFs on their surface and exposed to the same magnet $(0.3 \mathrm{~T})$ were exploited. We found that, in terms of the fraction of cells collected to the magnet and the time scale employed, the results obtained by using the MFNBs are far more significant for a cell separation application. With single IONPs functionalized with OTFs in the previously developed system, in $24 \mathrm{~h}$ of incubation, we could increase the ratio of the number of cells attracted to the magnet to the number of cells in a control sample (no magnet applied) to about only 2.5. ${ }^{[10]}$ This effect is due mainly to the intrinsic features of the system, which are clusters of IONPs in the nanostructure of this work and single IONPs in the nanostructures of our previous work. ${ }^{[10]}$

A complementary cell viability assay, based on the MTT test (see Figure $3 b$ ), allowed us to show that the cytotoxicity of the MFNBs on the cells was less then $20 \%$ for iron concentrations equal to $16 \mu \mathrm{g} \cdot \mathrm{mL}^{-1}$, while it reached $40 \%$ for the highest iron concentration of $65 \mu \mathrm{g} \cdot \mathrm{mL}^{-1}$. This suggested that with the isolated cells there is no need to detach the MFNBs because negligible cell toxicity was observed. The toxicity found with the beads was actually slightly higher than that of single IONPs functionalized with OTFs of the previous work. ${ }^{[10]}$ These results are likely due to a different surface functionalization of the two materials. Both types of samples were coated with the same polymer, but the IONPs had poly(ethylene glycol) molecules on their outer surface. ${ }^{[17]}$ In general, this reduced the degree 
of cell internalization of the IONPs and thus likely their "apparent" toxicity (as also confirmed by preliminary results of IONP internalization, not shown here). The uptake of the MFNBs was confirmed also via confocal imaging, by performing scans along the $z$-axis of doped cells (Figure 3c, see Supplementary Figure S15), which showed clearly the localization of the beads within the cytoplasm inside perinuclear vesicles. A deeper study on the fate of the MFNBs once taken up by the cell as a function of time is currently in progress. Preliminary experiments indicate that the cells once doped with the beads can respond to the magnet even if the separation experiment is performed 48 or $72 \mathrm{~h}$ after the doping (see Supplementary Figure S16).

\section{Conclusion}

We have demonstrated that magnetic beads can be prepared by wrapping IONPs within a polymer shell. Suitable choice of solvent allowed us to control the bead size, the number of IONPs encapsulated per bead (as well as their distribution in the bead), and consequently the magnetic features of the beads. This included the time needed to attract them to a magnet, which, depending on their size, might be exploited in both in vitro and in vivo applications. The magnetic beads have been further equipped with fluorescent tags based on OTF dyes, making them suitable for simultaneous cell separation and multiplexing detection. Preliminary cell separation experiments have shown the viability of our system for isolating cells in suspension within only $30 \mathrm{~min}$, with a separation efficiency of nearly $100 \%$.

Acknowledgements: This work was supported in part by the Italian Ministry of Research under FIRB Grants RBN01KJHT-007 and RBIN048TSE, and in part by the Italian Institute of Technology.
Received: April 23, 2009; Accepted: April 28, 2009; Published online: July 10, 2009; DOI: 10.1002/mabi.200900154

Keywords: cell sorting; fluorescent detection; magnetic separation; multifunctional platform; nanocrystals; nanostructures

[1] E. O. Song, G. P. Wang, H. Y. Xie, Z. L. Zhang, J. Hu, J. Peng, D. C. Wu, Y. B. Shi, D. W. Pang, Clin. Chem. 2007, 53, 2177.

[2] J. Yang, S. R. Dave, X. H. Gao, J. Am. Chem. Soc. 2008, 130, 5286.

[3] J. Guo, C. C. Wang, W. Y. Mao, W. L. Yang, C. J. Liu, J. Y. Chen, Nanotechnology 2008, 19, 15605.

[4] H. Y. Xie, C. Zuo, Y. Liu, Z. L. Zhang, D. W. Pang, X. L. Li, J. P. Gong, C. Dickinson, W. Z. Zhou, Small 2005, 1, 506.

[5] J. H. Park, G. von Maltzahn, E. Ruoslahti, S. N. Bhatia, M. J. Sailor, Angew. Chem. Int. Ed. 2008, 47, 7284.

[6] S. A. Corr, Y. P. Rakovich, Y. K. Gun'ko, Nanoscale Res. Lett. 2008, 3, 87

[7] A. Quarta, R. Di Corato, L. Manna, A. Ragusa, T. Pellegrino, IEEE Trans. Nanobiosci. 2007, 6, 298.

[8] G. Barbarella, M. Zambianchi, A. Ventola, E. Fabiano, F. Della Sala, G. Gigli, M. Anni, A. Bolognesi, L. Polito, M. Naldi, M. Capobianco, Bioconjugate Chem. 2006, 17, 58.

[9] G. Barbarella, L. Favaretto, G. Sotgiu, M. Zambianchi, V. Fattori, M. Cocchi, F. Cacialli, G. Gigli, R. Cingolani, Adv. Mater. 1999, 11, 1375

[10] A. Quarta, R. Di Corato, L. Manna, S. Argentiere, R. Cingolani, G. Barbarella, T. Pellegrino, J. Am. Chem. Soc. 2008, 130, 10545.

[11] J. P. Ge, Y. X. Hu, Y. D. Yin, Angew. Chem. Int. Ed. 2007, 46, 7428.

[12] J. P. Ge, Y. P. Hu, T. R. Zhang, T. Huynh, Y. D. Yin, Langmuir 2008, 24, 3671.

[13] J. Fresnais, J. F. Berret, B. F. Petesic, O. Sandre, R. Perzynski, Adv. Mater. 2008, 20, 3877.

[14] R. Di Corato, A. Quarta, P. Piacenza, A. Ragusa, A. Figuerola, R. Buonsanti, R. Cingolani, L. Manna, T. Pellegrino, J. Mater. Chem. 2008, 18, 1991.

[15] G. Barbarella, M. Zambianchi, O. Pudova, V. Paladini, A. Ventola, F. Cipriani, G. Gigli, R. Cingolani, G. Citro, J. Am. Chem. Soc. 2001, 123, 11600.

[16] S. H. Sun, H. Zeng, J. Am. Chem. Soc. 2002, 124, 8204.

[17] R. A. Sperling, T. Pellegrino, J. K. Li, W. H. Chang, W. J. Parak, Adv. Funct. Mater. 2006, 16, 943. 